\title{
Invariant Measure of the Infinite Dimensional Rotation Group
}

By

Yasuo YAMASAKI

\section{§1. Preliminary Discussions}

Let $H$ be a real Hilbert space, and $O(H)$ be its rotation group, namely the group of all orthogonal operators of $H$. We intend to construct an invariant probability measure on $O(H)$. However, such measure does not exist as proved below.

Let $\left\{e_{n}\right\}$ be a CONS (=complete orthonormal system) of $H$, and $\alpha_{n m}$ be the mean of $\left(U e_{n}, e_{m}\right)^{2}$ with respect to a measure $\mu$ on $O(H)$, namely

$$
\alpha_{n m}=\int\left(U e_{n}, e_{m}\right)^{2} d \mu(U)
$$

If $\mu$ is left invariant, for any orthogonal operator $U_{0}$ we have

$$
\alpha_{n m}=\int\left(U_{0} U e_{n}, e_{m}\right)^{2} d \mu(U)=\int\left(U e_{n}, U_{0}^{*} e_{m}\right)^{2} d \mu(U),
$$

hence especially we have $\alpha_{n m}=\alpha_{n 1}$, therefore $\alpha_{n m}$ does not depend on $m$. Thus $\sum_{m=1}^{\infty} \alpha_{n m}$ must be 0 or $\infty$, according to $\alpha_{n 1}=0$ or $>0$.

On the other hand, for any $U \in O(H)$ we have $\sum_{m=1}^{\infty}\left(U e_{n}, e_{m}\right)^{2}=\left\|U e_{n}\right\|^{2}$ $=1$, so that integrating both hand sides with respect to $\mu$ we get $\sum_{m=1}^{\infty} \alpha_{n m}=1$, which is impossible. Therefore, left invariant measure can not exist on $O(H)$.

In a similar but rather complicated way, we can prove that $O(H)$ invariant measure does not exist on $\mathcal{L}(H)$, the set of all linear continuous

Received October 28, 1971. 
operators of $H$, except the Dirac measure concentrated on zero operator. The latter is trivially $O(H)$-invariant.

Hence, in order to construct an $O(H)$-invariant measure, we must extend the group $O(H)$ to some space which is larger enough than $\ell(H)$.

\section{§2. Gaussian Measure}

If we fix a CONS $\left\{e_{n}\right\}$ of $H, H$ can be identified with $\left(l^{2}\right)$, the space of all square summable sequences. The space $\left(l^{2}\right)$ containes $R_{0}^{\infty}$, and is contained in $R^{\infty}$, where $R_{0}^{\infty}=\left\{\left.\left(x_{1}, x_{2}, \ldots\right)\right|^{\exists} n, x_{n+1}=x_{n+2}=\cdots=0\right\}$ and $R^{\infty}$ is the space of all sequences.

Any linear operator $A$ from $R_{0}^{\infty}$ to $R^{\infty}$ is determined uniquely from the double sequence $\left(a_{n m}\right)$ by the relation:

$$
e_{n}=(0,0, \ldots, 1,0, \ldots) \stackrel{A}{\longrightarrow}\left(a_{n 1}, a_{n 2}, \ldots, a_{n m}, \ldots\right)
$$

Thus the set $\mathcal{L}\left(R_{0}^{\infty}, R^{\infty}\right)$ of all linear operators from $R_{0}^{\infty}$ to $R^{\infty}$ can be identified with $R^{\infty \infty}$, the space of all double sequences. However, since $R^{\infty \infty}$ is isomorphic with $R^{\infty}$, we can consider Gaussian measure with variance 1 on the space $R^{\infty \infty}$. This is a measure on $R^{\infty \infty}$ such that for any finite number of $a_{n_{1} m_{1}}, \ldots, a_{n_{k} m_{k}}$, their joint distribution becomes $k$-dimensional Gaussian measure with unit variance.

I st $O_{0}(H)$ be the group of such orthogonal operators of $H$ that keep invariant $R_{0}^{\infty}$. If $U \in O_{0}(H), U$ can be considered as an operator on $R_{0}^{\infty}$, and its adjoint operator $U^{*}$ can be considered as an operator on $R^{\infty}$. In this sense, the above constructed Gaussian measure is $O_{0}(H)$-invariant as proved below.

If $A \in \mathcal{L}\left(R_{0}^{\infty}, R^{\infty}\right)$ corresponds to $\left(a_{n m}\right)$, for any $U \in O_{0}(H) \quad A U$ corresponds to $\left(\sum_{k=1}^{\infty} u_{n k} a_{m k}\right)$ and $U^{*} A$ corresponds to $\left(\sum_{k=1}^{\infty} a_{n k} u_{m k}\right)$ where $U e_{n}=\sum_{k=1}^{\infty} u_{n k} e_{k}$ which is actually a finite sum. Thus, multiplication of $U$ from right or of $U^{*}$ from left induces a rotation of row or column vectors of $\left(a_{n m}\right)$. Since Gaussian measure is rotationally invariant, such transformation keeps it invariant. 
Hereafter, Gaussian measure with variance 1 on $R^{\infty \infty}$ will be denoted with $g$.

\section{§ 3. Other Invariant Measures}

Consider a triplet $E_{1} \subset H \subset E_{2}$ of separable Hilbert spaces where $E_{1}$ is continuously and densely imbedded in $H$ while $H$ is continuously and densely imbedded in $E_{2}$. Let $\mathcal{L}\left(E_{1}, E_{2}\right)$ be the set of all linear continuous operators from $E_{1}$ to $E_{2}$. It contains $\mathcal{L}(H)=\mathcal{L}(H, H)$.

Let $O_{1}(H)$ be the group of such orthogonal operators of $H$ that keep $E_{1}$ invariant and act homeomorphically on $E_{1}$, and $O_{2}(H)$ be the group of such orthogonal operators of $H$ that can be extended to a homeomorphic operator on $E_{2}$. We intend to construct a measure on $\mathcal{L}\left(E_{1}, E_{2}\right)$ which is right invariant with respect to $O_{1}(H)$ and left invariant with respect to $\mathrm{O}_{2}(H)$.

Let $\left\{e_{n}\right\}$ be a CONS of $H$ such that $e_{n} \in E_{1}$ for any $n$. Identifying $H$ with $H^{*}$ (=the dual space of $H$ ), the space $E_{2}^{*}$ can be continuously and densely imbedded in $H$. Let $\left\{\boldsymbol{e}_{n}^{\prime}\right\}$ be another CONS of $H$ such that $e_{n}^{\prime} \in E_{2}^{*}$ for any $n$.

Now, for any $A \in \mathcal{L}\left(E_{1}, E_{2}\right), A e_{n}$ belongs to $E_{2}$. So that the mapping $A \rightarrow A e_{n}$ is a mapping from $\mathcal{L}\left(E_{1}, E_{2}\right)$ to $E_{2}$. Therefore any given measure $\mu$ on $\mathcal{L}\left(E_{1}, E_{2}\right)$ induces a measure $\mu_{n}$ on $E_{2}$. Namely, for any measurable set $B$ (=the set which belongs to the smallest $\sigma$-field which contains all Borel cylinders) of $E_{2}$,

$$
\mu_{n}(B)=\mu\left(\left\{A ; A e_{n} \in B\right\}\right) .
$$

(We suppose that $\mu$ is defined on the smallest $\sigma$-field of $\mathcal{L}\left(E_{1}, E_{2}\right)$ which makes $(A x, \xi)$ measurable for any $x \in E_{1}, \xi \in E_{2}^{*}$.)

For any $U \in O_{2}(H), U A e_{n} \in B$ is equivalent with $A e_{n} \in U^{-1} B$. Therefore if $\mu$ is left $O_{2}(H)$-invariant, $\mu_{n}$ is also an $O_{2}(H)$-invariant measure on $E_{2}$. Hence, $\mu_{n}$ must be a superposition of Gaussian measures with different variances and $E_{2}$ must be a nuclear extension of $H^{[1][2]}$ (Except the trivial case of Dirac measure.) 
On the other hand, for any $A \in \mathcal{L}\left(E_{1}, E_{2}\right), A^{*} e_{n}^{\prime}$ belongs to $E_{1}^{*}$. Hence, from the measure $\mu$ the mapping $A \rightarrow A^{*} e_{n}^{\prime}$ induces a measure $\mu_{n}^{\prime}$ on $E_{1}^{*}$. For any $U \in O_{1}(H),(A U)^{*} e_{n}^{\prime} \in B$ is equivalent with $A^{*} e_{n}^{\prime}$ $\in U^{*-1} B$. Therefore if $\mu$ is right $O_{1}(H)$-invariant, $\mu_{n}^{\prime}$ is also an $O_{1}(H)$ invariant measure on $E_{1}^{*}$. Hence, $\mu_{n}^{\prime}$ must be a superposition of Gaussian measures with different variances and $E_{1}$ must be nuclearly imbedded in $H$.

Theorem. If any infinite dimensional rotationally invariant measure exists on $\mathcal{L}\left(E_{1}, E_{2}\right)$ (except Dirac measure), $E_{2}$ must be a nuclear extension of $H$, and $E_{1}$ must be nuclearly imbedded in $H$.

The converse is also true.

We shall prove the converse. Let $E_{1} \subset H \subset E_{2}$ be a nuclearly imbedded triplet. We choose CONS $\left\{e_{n}\right\}$ of $H$ in $E_{1}$ and $\left\{e_{n}^{\prime}\right\}$ in $E_{2}^{*}$.

Any $A \in \mathscr{L}\left(E_{1}, E_{2}\right)$ is uniquely determined from the double sequence $\left(a_{n m}\right)$ by the relation $a_{n m}=\left(A e_{n}, e_{m}^{\prime}\right)$. Hence $\mathcal{L}\left(E_{1}, E_{2}\right)$ can be identified with a subspace of $R^{\infty \infty}$. Consider the Gaussian measure $g$ on $R^{\infty \infty}$. If $g\left(\mathcal{L}\left(E_{1}, E_{2}\right)\right)=1, g$ can be identified with a measure on $\mathcal{L}\left(E_{1}, E_{2}\right)$. Right $O_{1}(H)$-invariance and left $O_{2}(H)$-invariance of this measure are easily checked.

Let $\chi\left(E_{1}, E_{2}\right)$ be the set of all Hilbert-Schmidt operators from $E_{1}$ to $E_{2}$, namely

$$
A \in \chi\left(E_{1}, E_{2}\right) \Leftrightarrow \sum_{n=1}^{\infty}\left\|A f_{n}\right\|_{E_{2}}^{2}<\infty \quad \text { for CONS }\left\{f_{n}\right\} \text { of } E_{1} .
$$

Since $\chi\left(E_{1}, E_{2}\right) \subset \mathcal{L}\left(E_{1}, E_{2}\right)$, it is sufficient to prove $g\left(\eta\left(E_{1}, E_{2}\right)\right)=1$.

Without loss of generality, we can suppose that $\left\{e_{n}\right\}$ is a common orthogonal system of $H$ and $E_{1}$ with $\left\|e_{n}\right\|_{E_{1}}=\alpha_{n}$, and $\left\{e_{n}^{\prime}\right\}$ is a common orthogonal system of $H$ and $E_{2}^{*}$ with $\left\|e_{n}^{\prime}\right\|_{E_{2}^{*}}=\beta_{n}$. From the assumption of nuclear imbedding, we get $\sum_{n=1}^{\infty} \frac{1}{\alpha_{n}^{2}}<\infty$ and $\sum_{n=1}^{\infty} \frac{1}{\beta_{n}^{2}}<\infty$. Now, $A \in \chi\left(E_{1}, E_{2}\right)$ is equivalent with $\sum_{n=1}^{\infty} \frac{1}{\alpha_{n}^{2}}\left\|A e_{n}\right\|_{E_{2}}^{2}<\infty$, hence with $\sum_{n, m=1}^{\infty} \frac{1}{\alpha_{n}^{2}}\left(A e_{n}\right.$, $\left.\frac{e_{m}^{\prime}}{\beta_{m}}\right)^{2}=\sum_{n, m=1}^{\infty} \frac{1}{\alpha_{n}^{2}} \frac{1}{\beta_{m}^{2}} a_{n m}^{2}<\infty$. Since $\sum_{n, m=1}^{\infty} \frac{1}{\alpha_{n}^{2}} \frac{1}{\beta_{m}^{2}}<\infty, \eta\left(E_{1}, E_{2}\right)$ is identical with a nuclear extension of $\left(l^{2}\right)_{2}$, the space of square summable double 
sequences. So that the Gaussian measure $g$ lies on $\chi\left(E_{1}, E_{2}\right)$, namely $g\left(\chi\left(E_{1}, E_{2}\right)\right)=1$.

\section{§4. Properties of the Gaussian Measure}

Gaussian measure $g$ on $\mathcal{L}\left(E_{1}, E_{2}\right)$ is right $O_{1}(H)$-invariant and left $\mathrm{O}_{2}(\mathrm{H})$-invariant. Moreover;

$\left.1^{\circ}\right) \quad g$ is right $O_{1}(H)$-ergodic and left $O_{2}(H)$-ergodic.

For the proof of left $O_{2}(H)$-ergodicity, we shall show that for any bounded measurable function $f(A)$, the relation $f(U A)=f(A)$ for any $U \in O_{2}(H)$ implies $f(A)=$ constant modulo $g$-null set.

Since we suppose that $g$ is defined on the smallest $\sigma$-field that makes $(A x, \xi)$ measurable for $x \in E_{1}$ and $\xi \in E_{2}^{*}$, any bounded measurable function $f(A)$ can be approximated with a tame function. Namely, for given $\varepsilon>0$ there exist finite number of $x_{1}, x_{2}, \ldots, x_{n} \in E_{1}$ and $\xi_{1}, \xi_{2}, \ldots$, $\xi_{n} \in E_{2}^{*}$ and a function $\varphi$ of $n$ real variables such that

$$
\int\left|f(A)-\varphi\left(\left(A x_{1}, \xi_{1}\right), \ldots,\left(A x_{n}, \xi_{n}\right)\right)\right|^{2} d g(A)<\varepsilon .
$$

Since $g$ is left $O_{2}(H)$-invariant, if $f(U A)=f(A)$ we have

$$
\int\left|f(A)-\varphi\left(\left(A x_{1}, U^{*} \xi_{1}\right), \ldots,\left(A x_{n}, U^{*} \xi_{n}\right)\right)\right|^{2} d g(A)<\varepsilon .
$$

Therefore we have

$$
\begin{aligned}
& \int \mid \varphi\left(\left(A x_{1}, \xi_{1}\right), \ldots,\left(A x_{n}, \xi_{n}\right)\right) \\
& \quad-\left.\varphi\left(\left(A x_{1}, U^{*} \xi_{1}\right), \ldots,\left(A x_{n}, U^{*} \xi_{n}\right)\right)\right|^{2} d g(A)<4 \varepsilon .
\end{aligned}
$$

If $U^{*} \xi_{1}, \ldots, U^{*} \xi_{n}$ are orthogonal with $\xi_{1}, \xi_{2}, \ldots, \xi_{n}$, the left hand side becomes

$$
2 \int\left|\varphi\left(\left(A x_{1}, \xi_{1}\right), \ldots,\left(A x_{n}, \xi_{n}\right)\right)-m\right|^{2} d g(A)
$$

where

$$
m=\int \varphi\left(\left(A x_{1}, \xi_{1}\right), \ldots,\left(A x_{n}, \xi_{n}\right)\right) d g(A)
$$


Hence, $\varphi\left(\left(A x_{1}, \xi_{1}\right), \ldots,\left(A x_{n}, \xi_{n}\right)\right)$ is approximated with a constant function, so that $f(A)$ is approximated with a constant function. Therefore, letting $\varepsilon \rightarrow 0, f(A)$ must be a constant function.

Right $O_{1}(H)$-ergodicity of $g$ is proved in a similar way.

$2^{\circ}$ ) Since the variance of any matrix element of $A$ is 1 , we have

$$
\int(A x, \xi)^{2} d g(A)=1 \quad \text { for } x \in E_{1},\|x\|=1 \quad \text { and } \quad \xi \in E_{2}^{*},\|\xi\|=1,
$$

$3^{\circ}$ ) If $x_{1}, x_{2}, \ldots, x_{n} \in E_{1}$ are mutually orthogonal in $H$, the distributions of $A x_{1}, A x_{2}, \ldots, A x_{n}$ are mutually independent. Namely,

$$
g\left(\left\{A ; A x_{1} \in B_{1}, \cdots, A x_{n} \in B_{n}\right\}\right)=\prod_{k=1}^{n} g\left(\left\{A ; A x_{k} \in B_{k}\right\}\right) .
$$

This result comes from the fact that using the matrix representation of $A$ for suitable CONS, $A x_{k}$ corresponds to $k$-th row vector.

$4^{\circ}$ ) Though the measure $g$ can not be constructed on $O(H)$, the orthogonality of $A$ is assured in the following sense. For any $x, y \in E_{1}$, we have

$$
\lim _{n \rightarrow \infty} \frac{1}{n} \sum_{k=1}^{n}\left(A x, e_{k}^{\prime}\right)\left(A y, e_{k}^{\prime}\right)=(x, y) \quad \text { for } g \text {-almost all } A \text {. }
$$

This can be regarded as the orthogonality in mean. The proof is obtained from the strong law of large numbers.

\section{§5. Uniqueness}

Let $E_{1} \subset H \subset E_{2}$ be a nuclearly imbedded triplet of separable Hilbert spaces, and $\mu$ be a right $O_{1}(H)$-invariant and left $O_{2}(H)$-invariant measure on $\mathcal{L}\left(E_{1}, E_{2}\right)$. Let $\left\{e_{n}\right\}$ and $\left\{e_{n}^{\prime}\right\}$ be CONS of $H$ contained in $E_{1}$ and $E_{2}^{*}$ respectively.

As mentioned in $\S 3$, the mapping $A \rightarrow A e_{n}$ induces a measure $\mu_{n}$ on $E_{2}$. Since $\mu_{n}$ is $O_{2}(H)$-invariant, it is a superposition of Gaussian measures with different variances. If we assume that $\mu$ is left $O_{2}(H)$ ergodic, then $\mu_{n}$ is also $O_{2}(H)$-ergodic so that $\mu_{n}$ must be a single Gaussian measure with some variance. Since $\mu$ is right $O_{1}(H)$-invariant, 
measures $\mu_{n}$ do not depend on $n$. Therefore they are the same Gaussian measure.

The variance is determined as 1 , if we assume that for any $x \in E_{1}$, $\|x\|=1$ and $\xi \in E_{2}^{*},\|\xi\|_{1}=1$

$$
\int(A x, \xi)^{2} d \mu(A)=1 .
$$

Moreover, assume that if $x_{1}, x_{2}, \ldots, x_{n} \in E_{1}$ are mutually orthogonal in $H$, the distributions of $A x_{1}, A x_{2}, \ldots, A x_{n}$ are mutually independent. Then, the joint distribution of $A e_{1}, A e_{2}, \ldots, A e_{n}$ is the direct product of the distributions of $A e_{k}$, which are the Gaussian measure with unit variance. Thus, $\mu$ must be identical with the measure $g$ on $\mathcal{L}\left(E_{1}, E_{2}\right)$.

Theorem. A measure $\mu$ on $\mathcal{L}\left(E_{1}, E_{2}\right)$ is determined uniquely as $g$ under the following three conditions.

(1) $\mu$ is right $O_{1}(H)$-invariant and left $O_{2}(H)$-invariant.

(2) $\mu$ is left $\mathrm{O}_{2}(\mathrm{H})$-ergodic.

or $(2)^{\prime} \mu$ is right $O_{1}(H)$-ergodic.

(3) For any $x \in E_{1},\|x\|=1$ and $\xi \in E_{2}^{*},\|\xi\|=1$, we have

$$
\int(A x, \xi)^{2} d \mu(A)=1 \text {. }
$$

Remark that the mutually independence of $A e_{1}, A e_{2}, \ldots, A e_{n}$ is not necessary for the uniqueness of the invariant measure. From the next section on, we shall prove the theorem.

\section{§6. Characteristic Function}

Consider the following characteristic function of $\mu$.

$$
\chi(T)=\int \exp \left[i \sum_{n, m} t_{n m}\left(A e_{n}, e_{m}^{\prime}\right)\right] d \mu(A),
$$

where $T=\left(t_{n m}\right)$ is a double sequence which vanishes except finite number of $(n, m)$.

If $\mu$ is right $O_{1}(H)$-invarinat and left $O_{2}(H)$-invariant, $\chi(T)$ is invariant under any transformation of the form $T \rightarrow U T V$, where $U$ and 
$V$ are orthogonal matrices which correspond to elements of $O_{1}(H)$ and $\mathrm{O}_{2}(\mathrm{H})$ respectively.

The relation: $\quad T \equiv T^{\prime} \Leftrightarrow{ }^{3} U, V ; T^{\prime}=U T V$ is evidently an equivalence relation, and $\chi(T)$ is constant on any equivalence class. We shall remark that $T$ is equivalent with $T^{\prime}$ if and only if the eigenvalues of $T^{*} T$ are identical with those of $T^{*} T^{\prime}$. Therefore any $T$ is equivalent with a diagonal matrix $\Delta$. Thus, we have

$$
\chi(T)=\chi(\Delta)=\int \exp \left[i \sum_{n} \lambda_{n}\left(A e_{n}, e_{n}^{\prime}\right)\right] d \mu(A),
$$

where $\lambda_{n}^{2}$ are the eigenvalues of $T^{*} T$.

Put $\chi(T)=\varphi\left(\lambda_{1}^{2}, \lambda_{2}^{2}, \ldots, \lambda_{n}^{2}, \ldots\right)$. If we prove that $\varphi\left(\alpha_{1}, \alpha_{2}, \ldots\right)$ $=\exp \left[-\frac{1}{2} \sum_{n} \alpha_{n}\right]$, then for any $T$ we have $x(T)=\exp \left[-\frac{1}{2} \sum_{n, m} t_{n m}^{2}\right]$ because $\sum_{n, m} t_{n m}^{2}=\operatorname{Tr}\left(T^{*} T\right)$, thus $\mu$ must be the Gaussian measure with unit variance, namely $\mu=g$.

In order to determine the function $\varphi(\alpha)$, we need a lemma.

Let $[0, \infty)^{\infty}$ be the set of all non-negative sequences, and $[0, \infty)_{0}^{\infty}$ be a subspace of $[0, \infty)^{\infty}$ such that

$$
\alpha=\left(\alpha_{n}\right) \in[0, \infty)_{0}^{\infty} \Leftrightarrow{ }^{3} n ; \alpha_{n+1}=\alpha_{n+2}=\cdots=0 .
$$

Definition. A real function $\varphi(\alpha)$ defined on $[0, \infty)_{0}^{\infty}$ is called completely monotonic if it satisfies;

$$
(-1)^{m} \Delta_{\alpha_{1}} \Delta_{\alpha_{2}} \cdots \Delta_{\alpha_{m}} \varphi(\alpha) \geqq 0
$$

for any $m=0,1,2, \ldots,{ }^{\forall} \alpha \in[0, \infty)_{0}^{\infty},{ }^{\forall} \alpha_{k} \in[0, \infty)_{0}^{\infty}$, where $\Delta_{\alpha_{1}} \varphi(\alpha)$ $=\varphi\left(\alpha+\alpha_{1}\right)-\varphi(\alpha)$.

Lemma (Infinite dimensional Bernstein's theorem). Let $\varphi(\alpha)$ be completely monotonic on $[0, \infty)_{0}^{\infty}$ and right continuous at $\alpha=0$. Then, there exists uniquely a finite measure $m(\beta)$ on $[0, \infty)^{\infty}$ such that

$$
\varphi(\alpha)=\int \exp \left[-\sum_{n=1}^{\infty} \alpha_{n} \beta_{n}\right] d m(\beta) \quad \text { for }{ }^{\forall} \alpha \in[0, \infty)_{0}^{\infty}
$$

The proof, omitted here, is obtained from the corresponding theorem 
for finite dimensional case.

For a while, we assume the complete monotonicity of $\varphi(\alpha)$, which we shall prove later. Then from the lemma, there exists a corresponding measure $m(\beta)$ on $[0, \infty)^{\infty}$.

As mentioned in $\S 5$, under the conditions (1), (2) and (3) of $\S 5$, the measure $\mu_{1}$ of $A e_{1}$ is a Gaussian measure with variance 1 . Therefore we have

$$
\int \exp \left[i \lambda_{1}\left(A e_{1}, e_{1}^{\prime}\right)\right] d \mu(A)=\int \exp \left[i \lambda_{1}\left(x, e_{1}^{\prime}\right)\right] d \mu_{1}(x)=e^{-\frac{1}{2} \lambda_{1}^{2}}
$$

thus $\varphi\left(\alpha_{1}, 0,0, \ldots, 0, \ldots\right)=e^{-\frac{1}{2} \alpha_{1}}$.

Comparing with the result of the lemma, this means $\beta_{1}=\frac{1}{2}$ for $m$ almost all $\beta$.

In the same way, we have $\beta_{2}=\beta_{3}=\cdots=\beta_{n}=\cdots=\frac{1}{2}$ for $m$-almost all $\beta$. Therefore we get

$$
\varphi(\alpha)=\exp \left[-\frac{1}{2} \sum_{n} \alpha_{n}\right]
$$

which was our final purpose.

\section{$\S 7$. Complete Monotonicity}

Let $R_{0}^{\infty \infty}$ be the set of infinite dimensional matrices which vanish except finite number of elements, and $x(T)=\varphi\left(\alpha_{1}, \alpha_{2}, \ldots, \alpha_{n}, \ldots\right)$ be a function on $R_{0}^{\infty \infty}$ where $\alpha_{k}$ are eigenvalues of $T^{*} T$.

A finite subset $\left\{T_{1}, T_{2}, \ldots, T_{n}\right\}$ of $R_{0}^{\infty \infty}$ is called admissible if $\left(T_{j}-T_{k}\right)^{*}\left(T_{j}-T_{k}\right)$ is diagonal for any $1 \leqq j, k \leqq n$.

Proposition. If $\varphi(\alpha)$ is positive definite for any admissible subset, then
a) $\varphi(\alpha) \geqq 0$ for ${ }^{\forall} \alpha \in[0, \infty)_{0}^{\infty}$,
b) $\Delta_{\alpha_{1}} \varphi(\alpha)$ is negative definite for any admissible subset. 
The complete monotonicity of $\varphi(\alpha)$ is easily derived from this proposition.

Proof of a). For $\alpha=\left(\alpha_{1}, \alpha_{2}, \ldots, \alpha_{m}, 0, \ldots\right)$, put $T_{k}=\left(t_{p q}^{(k)}\right)$ where $t_{p q}^{(k)}=\sqrt{\alpha_{q}} \delta_{p-k m, q}$. Then $\left(T_{j}-T_{k}\right) *\left(T_{j}-T_{k}\right)$ is a diagonal matrix whose $(p, p)$ element is $2 \alpha_{p}$. From the positive definiteness, we have

$$
0 \leqq \sum_{j, k} x\left(T_{j}-T_{k}\right)=n \varphi(0)+n(n-1) \varphi(2 \alpha),
$$

hence $\varphi(2 \alpha) \geqq-\frac{1}{n-1} \varphi(0)$. Letting $n \rightarrow \infty$, we have $\varphi(2 \alpha) \geqq 0$.

Proof of b). We shall prove that for any admissible set $\left\{T_{k}\right\}$ and any complex numbers $\left\{\lambda_{k}\right\}$, we have $\sum \lambda_{j} \bar{\lambda}_{k} \Delta_{\alpha_{1}} \chi\left(T_{j}-T_{k}\right) \leqq 0$.

We can find such $T_{0}$ that satisfies $T_{0} * T_{k}=T_{k} * T_{0}=0$ for any $1 \leqq k \leqq n$, and $T_{0} * T_{0}$ is a diagonal matrix whose diagonal elements are $\alpha_{1}=\left(\alpha_{1}^{(1)}, \alpha_{2}^{(1)}, \ldots\right)$.

Put

$$
S_{k}=T_{k}, \quad \lambda_{k}^{\prime}=\lambda_{k} \quad \text { for } 1 \leqq k \leqq n
$$

and

$$
S_{k}=T_{k-n}+T_{0}, \quad \lambda_{k}^{\prime}=-\lambda_{k-n} \quad \text { for } n+1 \leqq k \leqq 2 n .
$$

Then, $\left\{S_{k}\right\}$ is an admissible set. From the positive definiteness of $\chi$ for $\left\{S_{k}\right\}$ we have

$$
2 \sum_{j, k=1}^{n} \lambda_{j} \bar{\lambda}_{k} \chi\left(T_{j}-T_{k}\right)-2 \sum_{j, k=1}^{n} \lambda_{j} \bar{\lambda}_{k} \chi\left(T_{j}-T_{k}-T_{0}\right) \geqq 0 .
$$

However, the diagonal elements of $\left(T_{j}-T_{k}-T_{0}\right) *\left(T_{j}-T_{k}-T_{0}\right)$ are evidently the corresponding diagonal elements of $\left(T_{j}-T_{k}\right)^{*}\left(T_{j}-T_{k}\right)$ plus $\alpha_{1}$. Thus, we have proved b).

\section{References}

[1] Minlos, R.A., Generalized random processes and their extension to measures, Trudy Moskov. Mat. Obšč. 8 (1959), 497-518 (in Russian).

[2] Umemura, Y., Measures on infinite dimensional vector spaces, Publ. RIMS Kyoto Univ. 1 (1965), 1-47. 